\title{
Existence and asymptotic expansion of the weak solution for a wave equation with nonlinear source containing nonlocal term
}

Nguyen Huu Nhan ${ }^{1,2}$, Le Thi Phuong Ngoc $^{3}$ and Nguyen Thanh Long ${ }^{2 *}$

\begin{tabular}{l}
\hline "Correspondence: \\
longnt2@gmail.com \\
2Department of Mathematics and \\
Computer Science, VNUHCM - \\
University of Science, 227 Nguyen \\
Van Cu Str., Dist. 5, Ho Chi Minh City, \\
Vietnam \\
Full list of author information is \\
available at the end of the article
\end{tabular}

available at the end of the article

\begin{abstract}
In this paper, we consider the Robin problem for a wave equation with nonlinear source containing nonlocal term. Using the Faedo-Galerkin method and the linearization method for nonlinear term, the existence and uniqueness of a weak solution are proved. An asymptotic expansion of high order in a small parameter of a weak solution is also discussed.
\end{abstract}

MSC: 35L20; 35L70; 35Q72

Keywords: Faedo-Galerkin method; linear recurrent sequence; Robin conditions; asymptotic expansion in a small parameter

\section{Introduction}

In this paper, we consider the Robin problem for a wave equation as follows:

$$
\begin{aligned}
& u_{t t}-u_{x x}=f\left(x, t, u(x, t), u\left(\eta_{1}, t\right), \ldots, u\left(\eta_{q}, t\right), u_{t}(x, t)\right), \quad 0<x<1,0<t<T, \\
& u_{x}(0, t)-h_{0} u(0, t)=u_{x}(1, t)+h_{1} u(1, t)=0, \\
& u(x, 0)=\tilde{u}_{0}(x), \quad u_{t}(x, 0)=\tilde{u}_{1}(x),
\end{aligned}
$$

where $f, \tilde{u}_{0}, \tilde{u}_{1}$ are given functions and $h_{0}, h_{1} \geq 0, \eta_{1}, \eta_{2}, \ldots, \eta_{q}$ are given constants with $h_{0}+h_{1}>0,0 \leq \eta_{1}<\eta_{2}<\cdots<\eta_{q} \leq 1$.

In some special cases, when the nonlinear term has various forms, the following nonlinear wave equation

$$
u_{t t}-\Delta u=F\left(x, t, u, u_{t}\right)
$$

where $\Delta$ is a Laplace operator, has been extensively studied by many authors, for example, we refer to [1-10] and the references given therein. In these works, many interesting results about existence, nonexistence, uniqueness, nonuniqueness, regularity, asymptotic behavior, asymptotic expansion, and decay of solutions were obtained.

In [2], Bergounioux considered Prob. (1.3)-(1.4) with the following boundary conditions:

$$
u_{x}(0, t)=P(t), \quad u_{x}(1, t)+K_{1} u(1, t)+\lambda_{1} u_{t}(1, t)=0
$$

(c) The Author(s) 2017. This article is distributed under the terms of the Creative Commons Attribution 4.0 International License (http://creativecommons.org/licenses/by/4.0/), which permits unrestricted use, distribution, and reproduction in any medium, provided you give appropriate credit to the original author(s) and the source, provide a link to the Creative Commons license, and indicate if changes were made. 
where $f, \tilde{u}_{0}, \tilde{u}_{1}$ are given functions, $K_{1}, \lambda_{1}$ are given constants and the unknown $u(x, t)$ and the unknown boundary value $P(t)$ satisfy the following Cauchy problem for an ordinary differential equation:

$$
\left\{\begin{array}{l}
P^{\prime \prime}(t)+\omega^{2} P(t)=h u_{t t}(0, t), \quad 0<t<T, \\
P(0)=P_{0}, \quad P^{\prime}(0)=P_{1}
\end{array}\right.
$$

where $\omega>0, h \geq 0, P_{0}, P_{1}$ are given constants and $K, \lambda$ are given nonnegative constants.

Prob. (1.4)-(1.6), with $F\left(x, t, u, u_{t}\right)=f(x, t)-K u-\lambda u_{t}$, describes the shock between a solid body and a linear viscoelastic bar resting on a viscoelastic base with linear elastic constraints at the side, constraints associated with a viscous frictional resistance.

With $F\left(x, t, u, u_{t}\right)=f(x, t)-h\left(u_{t}\right)$, Jokhadze, in [4], considered existence, uniqueness, and nonuniqueness, and nonexistence of a global classical solution for wave equations with nonlinear damping term.

In [5], the authors established the unique existence, stability, and asymptotic expansion of Prob. (1.3)-(1.4) with the nonlocal boundary conditions

$$
\left\{\begin{array}{l}
u_{x}(0, t)=g_{0}(t)+\int_{0}^{t} k_{0}(t-s) u(0, s) d s \\
-u_{x}(1, t)=g_{1}(t)+\int_{0}^{t} k_{1}(t-s) u(1, s) d s
\end{array}\right.
$$

where $F\left(x, t, u, u_{t}\right)=-\lambda u_{t}-f(u)$, with $\lambda$ is a given constant and $f, g_{0}, g_{1}, k_{0}, k_{1}$ are given functions. The existence and exponential decay for a nonlinear wave equation with a nonlocal boundary condition were also proved in [9].

Beilin, see [1], investigated the existence and uniqueness of a generalized solution for the following wave equation with an integral nonlocal condition:

$$
\left\{\begin{array}{l}
u_{t t}-\Delta u+c(x, t) u=f(x, t), \quad(x, t) \in \Omega \times(0, T), \\
\frac{\partial u}{\partial \eta}+\int_{0}^{t} \int_{\Omega} k(x, \xi, \tau) u(\xi, \tau) d \xi d \tau=0, \quad(x, t) \in \partial \Omega \times(0, T), \\
u(x, 0)=\tilde{u}_{0}(x), \quad u_{t}(x, 0)=\tilde{u}_{1}(x), \quad x \in \Omega,
\end{array}\right.
$$

where $\Omega$ is a bounded domain in $\mathbb{R}^{N}$ with a smooth boundary, $\eta$ is the unit outward normal on $\partial \Omega, f, \tilde{u}_{0}, \tilde{u}_{1}, k(x, \xi, \tau)$ are given functions. Nonlocal conditions come up when values of the function on the boundary are connected to values inside the domain. There are various types of nonlocal boundary conditions of integral form for hyperbolic, parabolic or elliptic equations, the ones were introduced in [1].

In recent years, some close forms of Eq. (1.4), with power-type nonlinearities containing integer power-type, fractional power-type or variable exponent, have been paid attention to by many researchers [3,11-13]. Benaissa and Messaoudi, in [3], considered the following problem:

$$
\left\{\begin{array}{l}
u_{t t}-\Delta u+g\left(u_{t}\right)+f(u)=0, \quad(x, t) \in \Omega \times(0, T), \\
u=0, \quad x \in \partial \Omega, t \geq 0, \\
u(x, 0)=\tilde{u}_{0}(x), \quad u_{t}(x, 0)=\tilde{u}_{1}(x), \quad x \in \Omega,
\end{array}\right.
$$

where $f(u)=-b|u|^{p-2} u, g\left(u_{t}\right)=a\left(1+\left|u_{t}\right|^{m-2} u_{t}\right), a, b>0, m, p>2$ and $\Omega$ is a bounded domain in $\mathbb{R}^{N}$ with a smooth boundary $\partial \Omega$. The authors showed that for suitably chosen 
initial data, (1.9) possesses a global weak solution, which decays exponentially even if $m>2$. The proof of global existence is based on the use of the potential well theory. In [11], Bhattarai proved the existence and stability of solitary-wave solutions of a system of 2-coupled nonlinear Schrödinger equations with power-type nonlinearities. By using variational methods, Repovš, in [13], established several existence results for Schrödingertype equations containing Laplace-type operators with variable exponent. Moreover, by using the fractional homotopy analysis transform method, Kumar [12] proposed a modified and simple algorithm for fractional modeling arising in unidirectional propagation of long wave in dispersive media.

In [14], the authors considered a one-dimensional nonlocal nonlinear strongly damped wave equation with dynamical boundary conditions. In other words, they looked to the following problem:

$$
\left\{\begin{array}{l}
u_{t t}-u_{x x}-\alpha u_{t x x}+\varepsilon f\left(u(1, t), \frac{u_{t}(1, t)}{\sqrt{\varepsilon}}\right)=0, \\
u(0, t)=0 \\
u_{t t}(1, t)=-\varepsilon\left[u_{x}(1, t)+\alpha u_{t x}(1, t)+r u_{t}(1, t)\right]-\varepsilon f\left(u(1, t), \frac{u_{t}(1, t)}{\sqrt{\varepsilon}}\right),
\end{array}\right.
$$

with $0<x<1, t>0, \alpha, r>0$, and $\varepsilon>0$. Prob. (1.10) models a spring-mass-damper system, where the term $\varepsilon f\left(u(1, t), \frac{u_{t}(1, t)}{\sqrt{\varepsilon}}\right)$ represents a control acceleration at $x=1$. By using the invariant manifold theory, the authors proved that for small values of the parameter $\varepsilon$, the solution of (1.10) attracted to a two-dimensional invariant manifold.

In [6], Long and Diem studied Prob. (1.3)-(1.4) with the nonlinear term of the form

$$
f\left(x, t, u, u_{x}, u_{t}\right)+\varepsilon g\left(x, t, u, u_{x}, u_{t}\right)
$$

associated with the mixed homogeneous boundary conditions

$$
u_{x}(0, t)-h_{0} u(0, t)=u_{x}(1, t)+h_{1} u(1, t)=0 .
$$

In the case of $f \in C^{2}\left([0,1] \times[0, \infty) \times \mathbb{R}^{3}\right)$ and $g \in C^{1}\left([0,1] \times[0, \infty) \times \mathbb{R}^{3}\right)$, an asymptotic expansion of order 2 in $\varepsilon$ is obtained for $\varepsilon$ sufficiently small.

We consider the following wave equation with the source containing nonlocal term:

$$
u_{t t}-u_{x x}=F\left(x, t, u(x, t), \int_{0}^{1} g(u(y, t)) d y\right), \quad 0<x<1,0<t<T,
$$

where $F, g$ are given continuous functions. Then, if the function $u(x, t)$ is continuous in $x$, the integral $\int_{0}^{1} g(u(y, t)) d y$ can be approximated by its Riemann sum

$$
\int_{0}^{1} g(u(y, t)) d y \approx \sum_{i=1}^{q} \frac{1}{q} g\left(u\left(\eta_{i}, t\right)\right),
$$

with $q$ is large enough and $\eta_{i}=i / q, i=1,2, \ldots, q$.

Therefore, the nonlinear term in (1.1) can be considered as an approximation of the one that appeared in (1.13) as follows:

$$
F\left(x, t, u(x, t), \int_{0}^{1} g(u(y, t)) d y\right) \approx F\left(x, t, u(x, t), \sum_{i=1}^{q} \frac{1}{q} g\left(u\left(\eta_{i}, t\right)\right)\right) .
$$


The approximation given in (1.15) and the aforementioned works lead to the ideas to study the existence and asymptotic expansion for the Robin problem for a wave equation with nonlinear source containing nonlocal term (1.1)-(1.3). The paper consists of four sections. In Section 2, we present some preliminaries. In Section 3, we associate with Prob. (1.1)-(1.3) a linear recurrent sequence which is bounded in a suitable space of functions. The existence of a local weak solution and the uniqueness are proved by using the FaedoGalerkin method and the weak compact method. In Section 4, we establish an asymptotic expansion of a weak solution $u_{\varepsilon}(x, t)$ of order $N+1$ in a small parameter $\varepsilon$ for the equation

$$
\begin{aligned}
u_{t t}-u_{x x}= & f\left(x, t, u(x, t), u\left(\eta_{1}, t\right), \ldots, u\left(\eta_{q}, t\right), u_{t}(x, t)\right) \\
& +\varepsilon f_{1}\left(x, t, u(x, t), u\left(\eta_{1}, t\right), \ldots, u\left(\eta_{q}, t\right), u_{t}(x, t)\right),
\end{aligned}
$$

$0<x<1,0<t<T$, associated with (1.2), (1.3). The results obtained here may be considered as a relative generalization of the results obtained in $[2,4-6,9]$, and [10].

\section{Preliminaries}

Put $\Omega=(0,1)$. We will omit the definitions of the usual function spaces and denote them by $L^{p}=L^{p}(\Omega), H^{m}=H^{m}(\Omega)$. Let $\langle\cdot, \cdot\rangle$ be either the scalar product in $L^{2}$ or the dual pairing of a continuous linear functional and an element of a function space. The notation $\|\cdot\|$ stands for the norm in $L^{2}$, and we denote by $\|\cdot\|_{X}$ the norm in the Banach space $X$. We call $X^{\prime}$ the dual space of $X$. We denote $L^{p}(0, T ; X), 1 \leq p \leq \infty$ the Banach space of real functions $u:(0, T) \rightarrow X$ measurable, such that $\|u\|_{L^{p}(0, T ; X)}<+\infty$, with

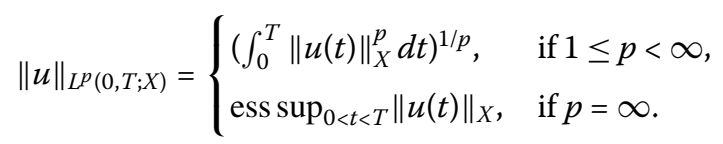

Let $u(t), u^{\prime}(t)=u_{t}(t)=\dot{u}(t), u^{\prime \prime}(t)=u_{t t}(t)=\ddot{u}(t), u_{x}(t)=\nabla u(t), u_{x x}(t)=\Delta u(t)$, denote $u(x, t), \frac{\partial u}{\partial t}(x, t), \frac{\partial^{2} u}{\partial t^{2}}(x, t), \frac{\partial u}{\partial x}(x, t), \frac{\partial^{2} u}{\partial x^{2}}(x, t)$, respectively.

With $f \in C^{k}\left([0,1] \times \mathbb{R}_{+} \times \mathbb{R}^{q+2}\right), f=f\left(x, t, y_{1}, \ldots, y_{q+2}\right)$, we put $D_{1} f=\frac{\partial f}{\partial x}, D_{2} f=\frac{\partial f}{\partial t}, D_{i+2} f=$ $\frac{\partial f}{\partial y_{i}}$ with $i=1, \ldots, q+2$, and $D^{\alpha} f=D_{1}^{\alpha_{1}} \cdots D_{q+4}^{\alpha_{q+4}} f, \alpha=\left(\alpha_{1}, \ldots, \alpha_{q+4}\right) \in \mathbb{Z}_{+}^{q+4},|\alpha|=\alpha_{1}+\cdots+$ $\alpha_{q+4}=k, D^{(0, \ldots, 0)} f=f$.

On $H^{1}$, we shall use the following norm:

$$
\|v\|_{H^{1}}=\left(\|v\|^{2}+\left\|v_{x}\right\|^{2}\right)^{1 / 2}
$$

We put

$$
a(u, v)=\int_{0}^{1} u_{x}(x) v_{x}(x) d x+h_{0} u(0) v(0)+h_{1} u(1) v(1), \quad u, v \in H^{1} .
$$

We have the following lemmas, the proofs of which are straightforward, hence we omit the details.

Lemma 2.1 ([15], Theorem 8.8, pp.212-213) The imbedding $H^{1} \hookrightarrow C^{0}(\bar{\Omega})$ is compact and

$$
\|v\|_{C^{0}(\bar{\Omega})} \leq \sqrt{2}\|v\|_{H^{1}} \quad \text { for all } v \in H^{1} .
$$


Lemma 2.2 Let $h_{0}, h_{1} \geq 0$, with $h_{0}+h_{1}>0$. Then the symmetric bilinear form a $(\cdot, \cdot)$ defined by (2.1) is continuous on $H^{1} \times H^{1}$ and coercive on $H^{1}$, i.e.,

(i) $|a(u, v)| \leq a_{1}\|u\|_{H^{1}}\|v\|_{H^{1}}$,

(ii) $a(v, v) \geq a_{0}\|v\|_{H^{1}}^{2}$,

for all $u, v \in H^{1}$, where $a_{1}=1+2 h_{0}+2 h_{1}$ and

$$
a_{0}=\frac{1}{4} \min \left\{1, \max \left\{h_{0}, h_{1}\right\}\right\} .
$$

Remark 2.1 It follows from (2.3) that on $H^{1}, v \longmapsto\|v\|_{H^{1}}, v \longmapsto\|v\|_{a}=\sqrt{a(v, v)}$ are two equivalent norms satisfying

$$
\sqrt{a_{0}}\|v\|_{H^{1}} \leq\|v\|_{a} \leq \sqrt{a_{1}}\|v\|_{H^{1}}, \quad \forall v \in H^{1}
$$

Lemma 2.3 Let $h_{0} \geq 0$. Then there exists the Hilbertorthonormal base $\left\{\widetilde{w}_{j}\right\}$ of $L^{2}$ consisting of the eigenfunctions $\widetilde{w}_{j}$ corresponding to the eigenvalue $\lambda_{j}$ such that

$$
\left\{\begin{array}{l}
0<\lambda_{1} \leq \lambda_{2} \leq \cdots \leq \lambda_{j} \leq \cdots, \quad \lim _{j \rightarrow+\infty} \lambda_{j}=+\infty \\
a\left(\widetilde{w}_{j}, v\right)=\lambda_{j}\left\langle\widetilde{w}_{j}, v\right\rangle \quad \text { for all } v \in H^{1}, j=1,2, \ldots
\end{array}\right.
$$

Furthermore, the sequence $\left\{\widetilde{w}_{j} / \sqrt{\lambda_{j}}\right\}$ is also a Hilbert orthonormal base of $H^{1}$ with respect to the scalar product a $(\cdot, \cdot)$.

On the other hand, we also have $\widetilde{w}_{j}$ satisfying the following boundary value problem:

$$
\left\{\begin{array}{l}
-\Delta \widetilde{w}_{j}=\lambda_{j} \widetilde{w}_{j}, \quad \text { in }(0,1), \\
\widetilde{w}_{j x}(0)-h_{0} \widetilde{w}_{j}(0)=\widetilde{w}_{j x}(1)+h_{1} \widetilde{w}_{j}(1)=0, \quad \widetilde{w}_{j} \in C^{\infty}(\bar{\Omega}) .
\end{array}\right.
$$

The proof of Lemma 2.3 can be found in ([16], p.87, Theorem 7.7), with $H=L^{2}$ and $V=H^{1}, a(\cdot, \cdot)$ as defined by $(2.1)$.

Remark 2.2 The weak formulation of the initial-boundary value problem (1.1)-(1.3) can be given in the following manner: Find $u \in \widetilde{W}=\left\{u \in L^{\infty}\left(0, T ; H^{2}\right): u_{t} \in L^{\infty}\left(0, T ; H^{1}\right), u_{t t} \in\right.$ $\left.L^{\infty}\left(0, T ; L^{2}\right)\right\}$ such that $u$ satisfies the following variational equation:

$$
\left\langle u_{t t}(t), w\right\rangle+a(u(t), w)=\left\langle f\left(\cdot, t, u(t), u\left(\eta_{1}, t\right), \ldots, u\left(\eta_{q}, t\right), u_{t}(t)\right), w\right\rangle
$$

for all $w \in H^{1}$, a.e., $t \in(0, T)$, together with the initial conditions

$$
u(0)=\tilde{u}_{0}, \quad u_{t}(0)=\tilde{u}_{1} .
$$

\section{The existence and uniqueness}

We make the following assumptions:

$\left(H_{1}\right) \quad\left(\tilde{u}_{0}, \tilde{u}_{1}\right) \in H^{2} \times H^{1}, \tilde{u}_{0 x}(0)-h_{0} \tilde{u}_{0}(0)=\tilde{u}_{0 x}(1)+h_{1} \tilde{u}_{0}(1)=0 ;$

$\left(H_{2}\right) f \in C^{1}\left([0,1] \times \mathbb{R}_{+} \times \mathbb{R}^{q+2}\right)$. 
Fix $T^{*}>0$. For each $M>0$ given, we set the constant $K_{M}(f)$ as follows:

$$
K_{M}(f)=\sum_{i=1}^{q+4} K_{0}\left(M, D_{i} f\right)
$$

where

$$
\left\{\begin{array}{l}
K_{0}(M, f)=\sup _{\left(x, t, y_{1}, \ldots, y_{q+2}\right) \in A_{1}(M)}\left|f\left(x, t, y_{1}, \ldots, y_{q+2}\right)\right| \\
A_{1}(M)=[0,1] \times\left[0, T^{*}\right] \times[-\sqrt{2} M, \sqrt{2} M]^{q+2}
\end{array}\right.
$$

For every $T \in\left(0, T^{*}\right]$ and $M>0$, we put

$$
\left\{\begin{aligned}
W(M, T)= & \left\{v \in L^{\infty}\left(0, T ; H^{2}\right): v_{t} \in L^{\infty}\left(0, T ; H^{1}\right), v_{t t} \in L^{2}\left(Q_{T}\right),\right. \\
& \text { with } \left.\max \left\{\|v\|_{L^{\infty}\left(0, T ; H^{2}\right)},\left\|v_{t}\right\|_{L^{\infty}\left(0, T ; H^{1}\right)},\left\|v_{t t}\right\|_{L^{2}\left(Q_{T}\right)}\right\} \leq M\right\} \\
W_{1}(M, T)= & \left\{v \in W(M, T): v_{t t} \in L^{\infty}\left(0, T ; L^{2}\right)\right\}
\end{aligned}\right.
$$

in which $Q_{T}=\Omega \times(0, T)$.

Now, we establish the recurrent sequence $\left\{u_{m}\right\}$. The first term is chosen as $u_{0} \equiv \tilde{u}_{0}$, suppose that

$$
u_{m-1} \in W_{1}(M, T)
$$

we associate Prob. (1.1)-(1.3) with the following problem.

Find $u_{m} \in W_{1}(M, T)(m \geq 1)$ satisfying the linear variational problem

$$
\left\{\begin{array}{l}
\left\langle u_{m}^{\prime \prime}(t), w\right\rangle+a\left(u_{m}(t), w\right)=\left\langle F_{m}(t), w\right\rangle, \quad \forall w \in H^{1}, \\
u_{m}(0)=\tilde{u}_{0}, \quad u_{m}^{\prime}(0)=\tilde{u}_{1}
\end{array}\right.
$$

where

$$
\begin{aligned}
F_{m}(x, t) & =f\left[u_{m-1}\right](x, t) \\
& =f\left(x, t, u_{m-1}(x, t), u_{m-1}\left(\eta_{1}, t\right), \ldots, u_{m-1}\left(\eta_{q}, t\right), u_{m-1}^{\prime}(x, t)\right) .
\end{aligned}
$$

Then we have the following theorem.

Theorem 3.1 Let $\left(H_{1}\right),\left(H_{2}\right)$ hold. Then there exist positive constants $M, T>0$ such that, for $u_{0} \equiv \tilde{u}_{0}$, there exists a recurrent sequence $\left\{u_{m}\right\} \subset W_{1}(M, T)$ defined by (3.1)-(3.3).

Proof The proof consists of several steps.

Step 1. The Faedo-Galerkin approximation (introduced by Lions [17]). Consider the basis $\left\{w_{j}\right\}$ for $H^{1}$ as in Lemma 2.3. Put

$$
u_{m}^{(k)}(t)=\sum_{j=1}^{k} c_{m j}^{(k)}(t) w_{j}
$$


where the coefficients $c_{m j}^{(k)}$ satisfy the system of linear differential equations

$$
\left\{\begin{array}{l}
\left\langle\ddot{u}_{m}^{(k)}(t), w_{j}\right\rangle+a\left(u_{m}^{(k)}(t), w_{j}\right)=\left\langle F_{m}(t), w_{j}\right\rangle, \quad 1 \leq j \leq k, \\
u_{m}^{(k)}(0)=\tilde{u}_{0 k}, \quad \dot{u}_{m}^{(k)}(0)=\tilde{u}_{1 k},
\end{array}\right.
$$

where $F_{m}(x, t)$ is defined as in (3.3) and

$$
\begin{cases}\tilde{u}_{0 k}=\sum_{j=1}^{k} \alpha_{j}^{(k)} w_{j} \rightarrow \tilde{u}_{0} & \text { strongly in } H^{2} \\ \tilde{u}_{1 k}=\sum_{j=1}^{k} \beta_{j}^{(k)} w_{j} \rightarrow \tilde{u}_{1} & \text { strongly in } H^{1}\end{cases}
$$

The system of equations (3.5) can be rewritten in the form

$$
\left\{\begin{array}{l}
\ddot{c}_{m j}^{(k)}(t)+\lambda_{j} c_{m j}^{(k)}(t)=\left\langle F_{m}(t), w_{j}\right\rangle, \\
c_{m}^{(k)}(0)=\alpha_{j}^{(k)}, \quad \dot{c}_{m j}^{(k)}(0)=\beta_{j}^{(k)}, \quad 1 \leq j \leq k .
\end{array}\right.
$$

It is not difficult to show that (3.7) has a unique solution $c_{m j}^{(k)}(t)$ in $[0, T]$ as follows:

$$
\begin{aligned}
c_{m j}^{(k)}(t)= & \alpha_{j}^{(k)} \cos \left(\sqrt{\lambda_{j}} t\right)+\beta_{j}^{(k)} \frac{\sin \left(\sqrt{\lambda_{j}} t\right)}{\sqrt{\lambda_{j}}} \\
& +\int_{0}^{t} \frac{\sin \left(\sqrt{\lambda_{j}}(t-s)\right)}{\sqrt{\lambda_{j}}}\left\langle F_{m}(s), w_{j}\right) d s, \\
& 0 \leq t \leq T, 1 \leq j \leq k .
\end{aligned}
$$

Therefore, (3.5) has a unique solution $u_{m}^{(k)}(t)$ in $[0, T]$.

Step 2. A priori estimates. First, for all $j=1, \ldots, k$, multiplying $(3.5)_{1}$ by $\dot{c}_{m j}^{(k)}(t)$, summing on $j$, and integrating with respect to the time variable from 0 to $t$, we have

$$
X_{m}^{(k)}(t)=X_{m}^{(k)}(0)+2 \int_{0}^{t}\left\langle F_{m}(s), \dot{u}_{m}^{(k)}(s)\right\rangle d s
$$

where

$$
X_{m}^{(k)}(t)=\left\|\dot{u}_{m}^{(k)}(t)\right\|^{2}+\left\|u_{m}^{(k)}(t)\right\|_{a}^{2} .
$$

Next, by replacing $w_{j}$ in $(3.5)_{1}$ by $-\frac{1}{\lambda_{j}} \Delta w_{j}$, we obtain that

$$
a\left(\ddot{u}_{m}^{(k)}(t), w_{j}\right)+\left\langle\Delta u_{m}^{(k)}(t), \Delta w_{j}\right\rangle=\left\langle F_{m}(t),-\Delta w_{j}\right\rangle, \quad 1 \leq j \leq k,
$$

similar to $(3.5)_{1}$, it gives

$$
\begin{aligned}
Y_{m}^{(k)}(t)= & Y_{m}^{(k)}(0)+2\left\langle F_{m}(0), \Delta \tilde{u}_{0 k}\right\rangle-2\left\langle F_{m}(t), \Delta u_{m}^{(k)}(t)\right\rangle \\
& +2 \int_{0}^{t}\left\langle F_{m}^{\prime}(s), \Delta u_{m}^{(k)}(s)\right\rangle d s
\end{aligned}
$$


Shan et al. Boundary Value Problems (2017) 2017:87

Page 8 of 20

where

$$
Y_{m}^{(k)}(t)=\left\|\dot{u}_{m}^{(k)}(t)\right\|_{a}^{2}+\left\|\Delta u_{m}^{(k)}(t)\right\|^{2}
$$

Put

$$
\begin{aligned}
S_{m}^{(k)}(t) & =X_{m}^{(k)}(t)+Y_{m}^{(k)}(t)+\int_{0}^{t}\left\|\ddot{u}_{m}^{(k)}(s)\right\|^{2} d s \\
& =\left\|\dot{u}_{m}^{(k)}(t)\right\|^{2}+\left\|\dot{u}_{m}^{(k)}(t)\right\|_{a}^{2}+\left\|u_{m}^{(k)}(t)\right\|_{a}^{2}+\left\|\Delta u_{m}^{(k)}(t)\right\|^{2}+\int_{0}^{t}\left\|\ddot{u}_{m}^{(k)}(s)\right\|^{2} d s,
\end{aligned}
$$

then we deduce from (3.9), (3.11), and (3.13) that

$$
\begin{aligned}
S_{m}^{(k)}(t)= & S_{m}^{(k)}(0)+2\left\langle F_{m}(0), \Delta \tilde{u}_{0 k}\right\rangle+2 \int_{0}^{t}\left\langle F_{m}(s), \dot{u}_{m}^{(k)}(s)\right\rangle d s \\
& -2\left\langle F_{m}(t), \Delta u_{m}^{(k)}(t)\right\rangle+2 \int_{0}^{t}\left\langle F_{m}^{\prime}(s), \Delta u_{m}^{(k)}(s)\right\rangle d s+\int_{0}^{t}\left\|\ddot{u}_{m}^{(k)}(s)\right\|^{2} d s \\
\equiv & S_{m}^{(k)}(0)+2\left\langle F_{m}(0), \Delta \tilde{u}_{0 k}\right\rangle+\sum_{j=1}^{4} I_{j} .
\end{aligned}
$$

We estimate all terms on the right-hand side of (3.14) as follows:

$$
\begin{aligned}
I_{1} & =2 \int_{0}^{t}\left\langle F_{m}(s), \dot{u}_{m}^{(k)}(s)\right\rangle d s \leq T K_{M}^{2}(f)+\int_{0}^{t} S_{m}^{(k)}(s) d s ; \\
I_{2} & =-2\left\langle F_{m}(t), \Delta u_{m}^{(k)}(t)\right\rangle \\
& \leq 4\left\|F_{m}(0)\right\|^{2}+4 T\left(\sqrt{T}[1+(q+1) \sqrt{2} M]^{2}+M\right)^{2} K_{M}^{2}(f)+\frac{1}{2} S_{m}^{(k)}(t) ; \\
I_{3} & =2 \int_{0}^{t}\left\langle F_{m}^{\prime}(s), \Delta u_{m}^{(k)}(s)\right\rangle d s \\
& \leq 2 T\left([1+(q+1) \sqrt{2} M]^{2}+M^{2}\right) K_{M}^{2}(f)+\int_{0}^{t} S_{m}^{(k)}(s) d s .
\end{aligned}
$$

We note that Eq. (3.5) 1 can be written as follows:

$$
\left\langle\ddot{u}_{m}^{(k)}(t), w_{j}\right\rangle-\left\langle\Delta u_{m}^{(k)}(t), w_{j}\right\rangle=\left\langle F_{m}(t), w_{j}\right\rangle, \quad 1 \leq j \leq k .
$$

Hence, after replacing $w_{j}$ with $\ddot{u}_{m}^{(k)}(t)$, we obtain

$$
\begin{aligned}
\left\|\ddot{u}_{m}^{(k)}(t)\right\|^{2} & =\left\langle\Delta u_{m}^{(k)}(t), \ddot{u}_{m}^{(k)}(t)\right\rangle+\left\langle F_{m}(t), \ddot{u}_{m}^{(k)}(t)\right\rangle \\
& \leq\left[\left\|\Delta u_{m}^{(k)}(t)\right\|+\left\|F_{m}(t)\right\|\right]\left\|\ddot{u}_{m}^{(k)}(t)\right\| \\
& \leq\left[\left\|\Delta u_{m}^{(k)}(t)\right\|+\left\|F_{m}(t)\right\|\right]^{2},
\end{aligned}
$$

So

$$
\begin{aligned}
I_{4} & =\int_{0}^{t}\left\|\ddot{u}_{m}^{(k)}(s)\right\|^{2} d s \leq 2 \int_{0}^{t}\left\|\Delta u_{m}^{(k)}(s)\right\|^{2} d s+2 \int_{0}^{t}\left\|F_{m}(s)\right\|^{2} d s \\
& \leq 2 \int_{0}^{t} S_{m}^{(k)}(s) d s+2 T K_{M}^{2}(f) .
\end{aligned}
$$


It follows from (3.14)-(3.17), (3.19) that

$$
S_{m}^{(k)}(t) \leq D_{0}^{(k)}\left(f, \tilde{u}_{0}, \tilde{u}_{1}, \tilde{u}_{0 k}, \tilde{u}_{1 k}\right)+D_{1}(M, T)+8 \int_{0}^{t} S_{m}^{(k)}(s) d s
$$

where

$$
\left\{\begin{array}{c}
D_{0}^{(k)}\left(f, \tilde{u}_{0}, \tilde{u}_{1}, \tilde{u}_{0 k}, \tilde{u}_{1 k}\right)=2 S_{m}^{(k)}(0)+4\left\langle F_{m}(0), \Delta \tilde{u}_{0 k}\right\rangle+8\left\|F_{m}(0)\right\|^{2} \\
D_{1}(M, T)=2\left[3+4\left(\sqrt{T}[1+(q+1) \sqrt{2} M]^{2}+M\right)^{2}\right. \\
\left.+2\left([1+(q+1) \sqrt{2} M]^{2}+M^{2}\right)\right] T K_{M}^{2}(f) .
\end{array}\right.
$$

By means of the convergence in (3.6), we can deduce the existence of a constant $M>0$ independent of $k$ and $m$ such that

$$
D_{0}^{(k)}\left(f, \tilde{u}_{0}, \tilde{u}_{1}, \tilde{u}_{0 k}, \tilde{u}_{1 k}\right) \leq \frac{1}{2} M^{2} \quad \text { for all } m, k \in \mathbb{N} \text {. }
$$

We choose $T \in\left(0, T^{*}\right]$ such that

$$
\left(\frac{1}{2} M^{2}+D_{1}(M, T)\right) e^{8 T} \leq M^{2}
$$

and

$$
k_{T}=\left(1+\frac{1}{\sqrt{a_{0}}}\right) \sqrt{2 T e^{T}}(q+1) K_{M}(f)<1 .
$$

Finally, it follows from (3.20), (3.22), and (3.23) that

$$
S_{m}^{(k)}(t) \leq M^{2} e^{-8 T}+8 \int_{0}^{t} S_{m}^{(k)}(s) d s
$$

By using Gronwall's lemma, we deduce from (3.25) that

$$
S_{m}^{(k)}(t) \leq M^{2} e^{-8 T} e^{8 t} \leq M^{2}
$$

for all $t \in[0, T]$, for all $m$ and $k$. Therefore, we have

$$
u_{m}^{(k)} \in W(M, T) \quad \text { for all } m \text { and } k
$$

Step 3. Limiting process. From (3.26), we deduce the existence of a subsequence of $\left\{u_{m}^{(k)}\right\}$, still so denoted, such that

$$
\left\{\begin{array}{l}
u_{m}^{(k)} \rightarrow u_{m} \quad \text { in } L^{\infty}\left(0, T ; H^{2}\right) \text { weak* } \\
\dot{u}_{m}^{(k)} \rightarrow u_{m}^{\prime} \text { in } L^{\infty}\left(0, T ; H^{1}\right) \text { weak }^{*} \\
\ddot{u}_{m}^{(k)} \rightarrow u_{m}^{\prime \prime} \text { in } L^{2}\left(Q_{T}\right) \text { weak, } \\
u_{m} \in W(M, T) .
\end{array}\right.
$$


Passing to limit in (3.5), we have $u_{m}$ satisfying (3.2), (3.3) in $L^{2}(0, T)$. On the other hand, it follows from $(3.2)_{1}$ and $(3.28)_{4}$ that $u_{m}^{\prime \prime}=\Delta u_{m}+F_{m} \in L^{\infty}\left(0, T ; L^{2}\right)$, hence $u_{m} \in W_{1}(M, T)$ and the proof of Theorem 3.1 is complete.

We use the result given in Theorem 3.1 and the compact imbedding theorems to prove the existence and uniqueness of a weak solution of Prob. (1.1)-(1.3). Hence, we get the main result in this section as follows.

Theorem 3.2 Let $\left(H_{1}\right),\left(H_{2}\right)$ hold. Then

(i) Prob. (1.1)-(1.3) has a unique weak solution $u \in W_{1}(M, T)$, where the constants $M>0$ and $T>0$ are chosen as in Theorem 3.1 .

(ii) The recurrent sequence $\left\{u_{m}\right\}$ defined by (3.1)-(3.3) converges to the solution u of Prob. (1.1)-(1.3) strongly in the space

$$
W_{1}(T)=\left\{v \in L^{\infty}\left(0, T ; H^{1}\right): v^{\prime} \in L^{\infty}\left(0, T ; L^{2}\right)\right\} .
$$

Furthermore, we also have the estimation

$$
\left\|u_{m}-u\right\|_{W_{1}(T)} \leq C_{T} k_{T}^{m} \quad \text { for all } m \in \mathbb{N}
$$

where the constant $k_{T} \in[0,1)$ is defined as in (3.24) and $C_{T}$ is a constant depending only on $T, h_{0}, h_{1}, f, \tilde{u}_{0}, \tilde{u}_{1}$, and $k_{T}$.

Proof (a) Existence of the solution. First, we note that $W_{1}(T)$ is a Banach space with respect to the norm (see Lions [17]).

$$
\|v\|_{W_{1}(T)}=\|v\|_{L^{\infty}\left(0, T ; H^{1}\right)}+\left\|v^{\prime}\right\|_{L^{\infty}\left(0, T ; L^{2}\right)}
$$

We shall prove that $\left\{u_{m}\right\}$ is a Cauchy sequence in $W_{1}(T)$. Let $w_{m}=u_{m+1}-u_{m}$. Then $w_{m}$ satisfies the variational problem

$$
\left\{\begin{array}{l}
\left\langle w_{m}^{\prime \prime}(t), w\right\rangle+a\left(w_{m}(t), w\right)=\left\langle F_{m+1}(t)-F_{m}(t), w\right\rangle, \quad \forall w \in H^{1}, \\
w_{m}(0)=w_{m}^{\prime}(0)=0 .
\end{array}\right.
$$

Taking $w=w_{m}^{\prime}$ in $(3.32)_{1}$, after integrating in $t$, we get

$$
Z_{m}(t)=2 \int_{0}^{t}\left\langle F_{m+1}(s)-F_{m}(s), w_{m}^{\prime}(s)\right\rangle d s,
$$

where

$$
Z_{m}(t)=\left\|w_{m}^{\prime}(t)\right\|^{2}+\left\|w_{m}(t)\right\|_{a}^{2}
$$

By $\left(H_{2}\right)$ it is clear that

$$
\begin{aligned}
\left\|F_{m+1}(t)-F_{m}(t)\right\| & \leq K_{M}(f)\left[\sqrt{2}(q+1)\left\|w_{m-1}(t)\right\|_{H^{1}}+\left\|w_{m-1}^{\prime}(t)\right\|\right] \\
& \leq \sqrt{2}(q+1) K_{M}(f)\left\|w_{m-1}\right\|_{W_{1}(T)} .
\end{aligned}
$$


Hence

$$
Z_{m}(t) \leq 2 T(q+1)^{2} K_{M}^{2}(f)\left\|w_{m-1}\right\|_{W_{1}(T)}^{2}+\int_{0}^{t} Z_{m}(s) d s .
$$

Using Gronwall's lemma, we deduce from (3.36) that

$$
\left\|w_{m}\right\|_{W_{1}(T)} \leq k_{T}\left\|w_{m-1}\right\|_{W_{1}(T)} \quad \forall m \in \mathbb{N},
$$

where $k_{T} \in(0,1)$ is defined as in (3.24), which implies that

$$
\left\|u_{m}-u_{m+p}\right\|_{W_{1}(T)} \leq\left\|u_{0}-u_{1}\right\|_{W_{1}(T)}\left(1-k_{T}\right)^{-1} k_{T}^{m} \quad \forall m, p \in \mathbb{N} .
$$

It follows that $\left\{u_{m}\right\}$ is a Cauchy sequence in $W_{1}(T)$. Then there exists $u \in W_{1}(T)$ such that

$$
u_{m} \rightarrow u \quad \text { strongly in } W_{1}(T) .
$$

Note that $u_{m} \in W_{1}(M, T)$, then there exists a subsequence $\left\{u_{m_{j}}\right\}$ of $\left\{u_{m}\right\}$ such that

$$
\left\{\begin{array}{l}
u_{m_{j}} \rightarrow u \quad \text { in } L^{\infty}\left(0, T ; H^{2}\right) \text { weak }^{*} \\
u_{m_{j}}^{\prime} \rightarrow u^{\prime} \quad \text { in } L^{\infty}\left(0, T ; H^{1}\right) \text { weak }^{*} \\
u_{m_{j}}^{\prime \prime} \rightarrow u^{\prime \prime} \quad \text { in } L^{2}\left(Q_{T}\right) \text { weak, } \\
u \in W(M, T)
\end{array}\right.
$$

We also note that

$$
\begin{aligned}
& \left\|F_{m}-f\left(\cdot, t, u(t), u\left(\eta_{1}, t\right), \ldots, u\left(\eta_{q}, t\right), u^{\prime}(t)\right)\right\|_{L^{\infty}\left(0, T ; L^{2}\right)} \\
& \quad \leq \sqrt{2}(q+1) K_{M}(f)\left\|u_{m-1}-u\right\|_{W_{1}(T)} .
\end{aligned}
$$

Hence, from (3.39) and (3.41), we obtain

$$
F_{m} \rightarrow f\left(\cdot, t, u(t), u\left(\eta_{1}, t\right), \ldots, u\left(\eta_{q}, t\right), u^{\prime}(t)\right) \quad \text { strongly in } L^{\infty}\left(0, T ; L^{2}\right) .
$$

Finally, passing to limit in (3.2)-(3.3) as $m=m_{j} \rightarrow \infty$, it implies from (3.39), (3.40) $)_{1,3}$, and (3.42) that there exists $u \in W(M, T)$ satisfying (2.6), (2.7).

On the other hand, from assumption $\left(H_{2}\right)$ we obtain from $(2.6),(3.40)_{4}$, and (3.42) that

$$
u^{\prime \prime}=u_{x x}+f\left(\cdot, t, u(t), u\left(\eta_{1}, t\right), \ldots, u\left(\eta_{q}, t\right), u^{\prime}(t)\right) \in L^{\infty}\left(0, T ; L^{2}\right)
$$

thus we have $u \in W_{1}(M, T)$. The existence proof is completed.

(b) Uniqueness of the solution. Let $u_{1}, u_{2} \in W_{1}(M, T)$ be two different weak solutions of Prob. (1.1)-(1.3). Then $u=u_{1}-u_{2}$ satisfies the variational problem

$$
\left\{\begin{array}{l}
\left\langle u^{\prime \prime}(t), w\right\rangle+a(u(t), w)=\left\langle F_{1}(t)-F_{2}(t), w\right\rangle, \quad \forall w \in H^{1}, \\
u(0)=u^{\prime}(0)=0,
\end{array}\right.
$$

where $F_{i}(x, t)=f\left(x, t, u_{i}(x, t), u_{i}\left(\eta_{1}, t\right), \ldots, u_{i}\left(\eta_{q}, t\right), u_{i}^{\prime}(x, t)\right), i=1,2$. 
We take $w=u^{\prime}$ in (3.44) $)_{1}$ and integrate in $t$ to get

$$
\left\|u^{\prime}(t)\right\|^{2}+\|u(t)\|_{a}^{2} \leq \sqrt{\frac{2}{a_{0}}}(q+1) K_{M}(f) \int_{0}^{t}\left(\left\|u^{\prime}(s)\right\|^{2}+\|u(s)\|_{a}^{2}\right) d s .
$$

Using Gronwall's lemma, it follows that $\left\|u^{\prime}(t)\right\|^{2}+\|u(t)\|_{a}^{2} \equiv 0$, i.e., $u_{1} \equiv u_{2}$.

So (i) is proved and (ii) follows. Theorem 3.2 is proved completely.

\section{Asymptotic expansion of the solution with respect to a small parameter}

In this section, let $\left(H_{1}\right),\left(H_{2}\right)$ hold. We make the following additional assumption:

$\left(H_{2}^{\prime}\right) f_{1} \in C^{1}\left([0,1] \times \mathbb{R}_{+} \times \mathbb{R}^{q+2}\right)$.

We consider the following perturbed problem, where $\varepsilon$ is a small parameter such that, $|\varepsilon| \leq 1$ :

$\left(P_{\varepsilon}\right)\left\{\begin{array}{l}u_{t t}-u_{x x}=F_{\varepsilon}[u](x, t), \quad 0<x<1,0<t<T, \\ u_{x}(0, t)-h_{0} u(0, t)=u_{x}(1, t)+h_{1} u(1, t)=0, \\ u(x, 0)=\tilde{u}_{0}(x), \quad u_{t}(x, 0)=\tilde{u}_{1}(x),\end{array}\right.$

where

$$
\left\{\begin{array}{l}
F_{\varepsilon}[u](x, t)=f[u](x, t)+\varepsilon f_{1}[u](x, t), \\
f[u](x, t)=f\left(x, t, u(x, t), u\left(\eta_{1}, t\right), \ldots, u\left(\eta_{q}, t\right), u^{\prime}(t)\right), \\
f_{1}[u](x, t)=f_{1}\left(x, t, u(x, t), u\left(\eta_{1}, t\right), \ldots, u\left(\eta_{q}, t\right), u^{\prime}(t)\right) .
\end{array}\right.
$$

First, we note that if the functions $f, f_{1}$ satisfy $\left(H_{2}\right),\left(H_{2}^{\prime}\right)$, then the a priori estimates of the Galerkin approximation sequence $\left\{u_{m}^{(k)}\right\}$ in the proof of Theorem 3.1 for Prob. (1.1)(1.3) corresponding to $f=F_{\varepsilon}[u],|\varepsilon| \leq 1$, satisfy $u_{m}^{(k)} \in W_{1}(M, T)$, where $M, T$ are constants independent of $\varepsilon$. We also note that the positive constants $M$ and $T$ are chosen as in (3.22)(3.23) with $\left|f\left(\cdot, 0, \tilde{u}_{0}, \tilde{u}_{0}\left(\eta_{1}\right), \ldots, \tilde{u}_{0}\left(\eta_{q}\right), \tilde{u}_{1}\right)\right|, K_{M}(f)$, stand for

$$
\begin{aligned}
& \left|f\left(\cdot, 0, \tilde{u}_{0}, \tilde{u}_{0}\left(\eta_{1}\right), \ldots, \tilde{u}_{0}\left(\eta_{q}\right), \tilde{u}_{1}\right)\right|+\left|f_{1}\left(\cdot, 0, \tilde{u}_{0}, \tilde{u}_{0}\left(\eta_{1}\right), \ldots, \tilde{u}_{0}\left(\eta_{q}\right), \tilde{u}_{1}\right)\right|, \\
& K_{M}(f)+K_{M}\left(f_{1}\right),
\end{aligned}
$$

respectively.

Hence, the limit $u_{\varepsilon}$ in suitable function spaces of the sequence $\left\{u_{m}^{(k)}\right\}$ as $k \rightarrow+\infty$, after $m \rightarrow+\infty$, is a unique weak solution of the problem $\left(P_{\varepsilon}\right)$ satisfying $u_{\varepsilon} \in W_{1}(M, T)$.

Then we can prove, in a manner similar to the proof of Theorem 3.2, that the limit $u_{0}$ in suitable function spaces of the family $\left\{u_{\varepsilon}\right\}$ as $\varepsilon \rightarrow 0$ is a unique weak solution of the problem $\left(P_{0}\right)$ (corresponding to $\varepsilon=0$ ) satisfying $u_{0} \in W_{1}(M, T)$.

We shall study the asymptotic expansion of the solution of the problem $\left(P_{\varepsilon}\right)$ with respect to a small parameter $\varepsilon$. 
We use the following notations. For a multi-index $\alpha=\left(\alpha_{1}, \ldots, \alpha_{N}\right) \in \mathbb{Z}_{+}^{N}$, and $x=$ $\left(x_{1}, \ldots, x_{N}\right) \in \mathbb{R}^{N}$, we put

$$
\left\{\begin{array}{l}
|\alpha|=\alpha_{1}+\cdots+\alpha_{N}, \quad \alpha !=\alpha_{1} ! \cdots \alpha_{N} ! \\
\alpha, \beta \in \mathbb{Z}_{+}^{N}, \quad \alpha \leq \beta \quad \Longleftrightarrow \quad \alpha_{i} \leq \beta_{i} \quad \forall i=1, \ldots, N, \\
x^{\alpha}=x_{1}^{\alpha_{1}} \cdots x_{N}^{\alpha_{N}} .
\end{array}\right.
$$

Next, we need the following lemma.

Lemma 4.1 Let $m, N \in \mathbb{N}$ and $x=\left(x_{1}, \ldots, x_{N}\right) \in \mathbb{R}^{N}, \varepsilon \in \mathbb{R}$. Then

$$
\left(\sum_{i=1}^{N} x_{i} \varepsilon^{i}\right)^{m}=\sum_{k=m}^{m N} P_{k}^{(m)}[N, x] \varepsilon^{k}
$$

where the coefficients $P_{k}^{(m)}[N, x], m \leq k \leq m N$ depending on $x=\left(x_{1}, \ldots, x_{N}\right)$ are defined by the formulas

$$
P_{k}^{(m)}[N, x]= \begin{cases}x_{k}, & 1 \leq k \leq N, m=1, \\ \sum_{\alpha \in A_{k}^{(m)}(N)} \frac{m !}{\alpha !} x^{\alpha}, & m \leq k \leq m N, m \geq 2,\end{cases}
$$

where $A_{k}^{(m)}(N)=\left\{\alpha \in \mathbb{Z}_{+}^{N}:|\alpha|=m, \sum_{i=1}^{N} i \alpha_{i}=k\right\}$.

The proof of Lemma 4.1 is easy, hence we omit the details.

Now, we assume that

$\left(H_{2}^{(N)}\right) f \in C^{N+1}\left([0,1] \times \mathbb{R}_{+} \times \mathbb{R}^{q+2}\right), f_{1} \in C^{N}\left([0,1] \times \mathbb{R}_{+} \times \mathbb{R}^{q+2}\right)$.

Let $u_{0}$ be a unique weak solution of the problem $\left(P_{0}\right)$ corresponding to $\varepsilon=0$, i.e.,

$\left(P_{0}\right)\left\{\begin{array}{l}u_{0}^{\prime \prime}-\Delta u_{0}=f\left[u_{0}\right] \equiv F_{0}, \quad 0<x<1,0<t<T, \\ u_{0 x}(0, t)-h_{0} u_{0}(0, t)=u_{0 x}(1, t)+h_{1} u_{0}(1, t)=0, \\ u_{0}(x, 0)=\tilde{u}_{0}(x), \quad u_{0}^{\prime}(x, 0)=\tilde{u}_{1}(x), \\ u_{0} \in W_{1}(M, T) .\end{array}\right.$

Let us consider the sequence of weak solutions $u_{k}, 1 \leq k \leq N$, defined by the following problems:

$$
\left(\tilde{P}_{k}\right)\left\{\begin{array}{l}
u_{k}^{\prime \prime}-\Delta u_{k}=F_{k}, \quad 0<x<1,0<t<T, \\
u_{k x}(0, t)-h_{0} u_{k}(0, t)=u_{k x}(1, t)+h_{1} u_{k}(1, t)=0, \\
u_{k}(x, 0)=u_{k}^{\prime}(x, 0)=0, \\
u_{k} \in W_{1}(M, T),
\end{array}\right.
$$

where $F_{k}, 1 \leq k \leq N$, are defined by the formulas

$$
F_{k}= \begin{cases}\bar{\Phi}_{1}[N, f]+f_{1}\left[u_{0}\right], & k=1, \\ \bar{\Phi}_{k}[N, f]+\bar{\Phi}_{k-1}\left[N-1, f_{1}\right], & 2 \leq k \leq N,\end{cases}
$$


with $\bar{\Phi}_{k}[N, f]=\bar{\Phi}_{k}\left[N, f, u_{0}, u_{0}^{\prime}, \vec{u}, \vec{u}^{\prime}\right], 0 \leq k \leq N$, are defined by the formulas

$$
\bar{\Phi}_{k}[N, f]= \begin{cases}f\left[u_{0}\right], & k=0, \\ \sum_{1 \leq|\gamma| \leq k} \frac{1}{\gamma !} D^{\gamma} f\left[u_{0}\right] \Psi_{k}\left[\gamma, N, \vec{u}, \vec{u}^{\prime}\right], & 1 \leq k \leq N,\end{cases}
$$

where

$$
\begin{aligned}
& \Psi_{k}\left[\gamma, N, \vec{u}, \vec{u}^{\prime}\right] \\
& =\sum_{\substack{\left(\beta_{1}, \ldots, \beta_{q+2}\right) \in \tilde{A}(\gamma, N), \beta_{1}+\cdots+\beta_{q+2}=k}} P_{\beta_{1}}^{\left(\gamma_{1}\right)}[N, \vec{u}(x, t)] P_{\beta_{2}}^{\left(\gamma_{2}\right)}\left[N, \vec{u}\left(\eta_{1}, t\right)\right] \cdots \\
& \quad \times P_{\beta_{q+1}}^{\left(\gamma_{q+1}\right)}\left[N, \vec{u}\left(\eta_{q}, t\right)\right] P_{\beta_{q+2}}^{\left(\gamma_{q+2}\right)}\left[N, \vec{u}^{\prime}(x, t)\right],
\end{aligned}
$$

with

$$
\left\{\begin{array}{l}
\widetilde{A}(\gamma, N)=\left\{\left(\beta_{1}, \ldots, \beta_{q+2}\right) \in \mathbb{Z}_{+}^{q+2}: \gamma_{i} \leq \beta_{i} \leq N \gamma_{i}, 1 \leq i \leq q+2\right\} \\
\gamma=\left(\gamma_{1}, \ldots, \gamma_{q+2}\right) \in \mathbb{Z}_{+}^{q+2}, \quad 1 \leq|\gamma| \leq N
\end{array}\right.
$$

and $\vec{u}(x, t)=\left(u_{1}(x, t), \ldots, u_{N}(x, t)\right), \vec{u}^{\prime}(x, t)=\left(\dot{u}_{1}(x, t), \ldots, \dot{u}_{N}(x, t)\right)$.

Then, we have the following theorem.

Theorem 4.2 Let $\left(H_{1}\right)$ and $\left(H_{2}^{(N)}\right)$ hold. Then there exist constants $M>0$ and $T>0$ such that, for every $\varepsilon \in[-1,1]$, the problem $\left(P_{\varepsilon}\right)$ has a unique weak solution $u_{\varepsilon} \in W_{1}(M, T)$ satisfying the asymptotic estimation up to order $N+1$ as follows:

$$
\left\|u_{\varepsilon}-\sum_{k=0}^{N} u_{k} \varepsilon^{k}\right\|_{W_{1}(T)} \leq C_{T}|\varepsilon|^{N+1}
$$

where the functions $u_{k}, 0 \leq k \leq N$, are the weak solutions of the problems $\left(P_{0}\right),\left(\tilde{P}_{k}\right), 1 \leq$ $k \leq N$, respectively, and $C_{T}$ is a constant depending only on $N, T, f, f_{1}, u_{k}, 0 \leq k \leq N$.

Remark 4.1 By the fact that it is very difficult to find $u_{\varepsilon}$ of the problem $\left(P_{\varepsilon}\right)$, we try to search the weak solutions $u_{k}, 0 \leq k \leq N$, of the problems $\left(P_{0}\right),\left(\tilde{P}_{k}\right)$. Clearly, they are found much more easily than $u_{\varepsilon}$ and $u_{\varepsilon}$ can be approximated by $u_{k}$ via (4.7).

In order to prove Theorem 4.2, we need the following lemmas.

Lemma 4.3 Let $\bar{\Phi}_{k}[N, f], 0 \leq k \leq N$, be the functions defined by formulas (4.4)-(4.6). Put $h=\sum_{k=0}^{N} u_{k} \varepsilon^{k}$, then we have

$$
f[h]=\sum_{k=0}^{N} \bar{\Phi}_{k}[N, f] \varepsilon^{k}+|\varepsilon|^{N+1} \hat{R}_{N}\left[f, u_{0}, \vec{u}, \vec{u}^{\prime}, \varepsilon\right],
$$

with $\left\|\hat{R}_{N}\left[f, u_{0}, \vec{u}, \vec{u}^{\prime}, \varepsilon\right]\right\|_{L^{\infty}\left(0, T ; L^{2}\right)} \leq C$, where $C$ is a constant depending only on $N, T, f, u_{k}$, $\dot{u}_{k}, 0 \leq k \leq N$. 
Proof of Lemma 4.3 (i) In the case of $N=1$, the proof of (4.8) is easy, hence we omit the details, which we only prove with $N \geq 2$. Put $h=u_{0}+\sum_{k=1}^{N} u_{k} \varepsilon^{k} \equiv u_{0}+h_{1}$, we rewrite as follows:

$$
\begin{aligned}
f[h] & (x, t) \\
= & f\left(x, t, h(x, t), h\left(\eta_{1}, t\right), \ldots, h\left(\eta_{q}, t\right), \dot{h}(x, t)\right) \\
= & f\left(x, t, u_{0}(x, t)+h_{1}(x, t), u_{0}\left(\eta_{1}, t\right)+h_{1}\left(\eta_{1}, t\right), \ldots,\right. \\
& \left.u_{0}\left(\eta_{q}, t\right)+h_{1}\left(\eta_{q}, t\right), \dot{u}_{0}(x, t)+\dot{h}_{1}(x, t)\right) .
\end{aligned}
$$

By using Taylor's expansion of the function $f[h]$ around the point

$$
\left[u_{0}\right] \equiv\left(x, t, u_{0}(x, t), u_{0}\left(\eta_{1}, t\right), \ldots, u_{0}\left(\eta_{q}, t\right), \dot{u}_{0}(x, t)\right)
$$

up to order $N+1$, we obtain

$$
\begin{aligned}
f[h]= & f\left[u_{0}\right]+\sum_{1 \leq|\gamma| \leq N} \frac{1}{\gamma !} D^{\gamma} f\left[u_{0}\right] h_{1}^{\gamma_{1}}(x, t) h_{1}^{\gamma_{2}}\left(\eta_{1}, t\right) \cdots \\
& \times h_{1}^{\gamma_{q+1}}\left(\eta_{q}, t\right) \dot{h}_{1}^{\gamma_{q+2}}(x, t)+R_{N}\left[f, u_{0}, h_{1}\right],
\end{aligned}
$$

where

$$
\begin{aligned}
& R_{N}\left[f, u_{0}, h_{1}\right] \\
& =\sum_{|\gamma|=N+1} \frac{N+1}{\gamma !} \int_{0}^{1}(1-\theta)^{N} D^{\gamma} f\left[u_{0}+\theta h_{1}\right] h_{1}^{\gamma_{1}}(x, t) h_{1}^{\gamma_{2}}\left(\eta_{1}, t\right) \cdots \\
& \times h_{1}^{\gamma_{q+1}}\left(\eta_{q}, t\right) \dot{h}_{1}^{\gamma_{q+2}}(x, t) d \theta \\
& =|\varepsilon|^{N+1} R_{N}^{(1)}\left[f, u_{0}, h_{1}, \varepsilon\right] \text {, } \\
& \gamma=\left(\gamma_{1}, \ldots, \gamma_{q+2}\right) \in \mathbb{Z}_{+}^{q+2}, \\
& |\gamma|=\gamma_{1}+\cdots+\gamma_{q+2}, \\
& \gamma !=\gamma_{1} ! \cdots \gamma_{q+2} ! \\
& D^{\gamma} f=D_{3}^{\gamma_{1}} D_{4}^{\gamma_{2}} \cdots D_{q+4}^{\gamma_{q+2}} f \text {, } \\
& D^{\gamma} f\left[u_{0}\right]=D^{\gamma} f\left(x, t, u_{0}(x, t), u_{0}\left(\eta_{1}, t\right), \ldots, u_{0}\left(\eta_{q}, t\right), \dot{u}_{0}(x, t)\right) .
\end{aligned}
$$

By formula (4.1), we get

$$
\begin{aligned}
& h_{1}^{\gamma_{1}}(x, t)=\left(\sum_{k=1}^{N} u_{k}(x, t) \varepsilon^{k}\right)^{\gamma_{1}}=\sum_{k=\gamma_{1}}^{N \gamma_{1}} P_{k}^{\left(\gamma_{1}\right)}[N, \vec{u}(x, t)] \varepsilon^{k}, \\
& h_{1}^{\gamma_{2}}\left(\eta_{1}, t\right)=\left(\sum_{k=1}^{N} u_{k}\left(\eta_{1}, t\right) \varepsilon^{k}\right)^{\gamma_{2}}=\sum_{k=\gamma_{2}}^{N \gamma_{2}} P_{k}^{\left(\gamma_{2}\right)}\left[N, \vec{u}\left(\eta_{1}, t\right)\right] \varepsilon^{k},
\end{aligned}
$$




$$
\begin{aligned}
& h_{1}^{\gamma_{q+1}}\left(\eta_{q}, t\right)=\left(\sum_{k=1}^{N} u_{k}\left(\eta_{q}, t\right) \varepsilon^{k}\right)^{\gamma_{q+1}}=\sum_{k=\gamma_{q+1}}^{N \gamma_{q+1}} P_{k}^{\left(\gamma_{q+1}\right)}\left[N, \vec{u}\left(\eta_{q}, t\right)\right] \varepsilon^{k}, \\
& \dot{h}_{1}^{\gamma_{q+2}}(x, t)=\left(\sum_{k=1}^{N} \dot{u}_{k}(x, t) \varepsilon^{k}\right)^{\gamma_{q+2}}=\sum_{k=\gamma_{q+2}}^{N \gamma_{q+2}} P_{k}^{\left(\gamma_{q+2}\right)}\left[N, \vec{u}^{\prime}(x, t)\right] \varepsilon^{k},
\end{aligned}
$$

where $\vec{u}(x, t)=\left(u_{1}(x, t), \ldots, u_{N}(x, t)\right), \vec{u}^{\prime}(x, t)=\left(\dot{u}_{1}(x, t), \ldots, \dot{u}_{N}(x, t)\right)$.

Hence, we deduce from (4.12), that

$$
\begin{aligned}
& h_{1}^{\gamma_{1}}(x, t) h_{1}^{\gamma_{2}}\left(\eta_{1}, t\right) \cdots h_{1}^{\gamma_{q+1}}\left(\eta_{q}, t\right) \dot{h}_{1}^{\gamma_{q+2}}(x, t) \\
& \quad=\sum_{k=|\gamma|}^{N} \Psi_{k}\left[\gamma, N, \vec{u}, \vec{u}^{\prime}\right] \varepsilon^{k}+\sum_{k=N+1}^{|\gamma| N} \Psi_{k}\left[\gamma, N, \vec{u}, \vec{u}^{\prime}\right] \varepsilon^{k},
\end{aligned}
$$

where

$$
\left\{\begin{array}{l}
\Psi_{k}\left[\gamma, N, \vec{u}, \vec{u}^{\prime}\right] \\
\quad=\sum_{\left(\beta_{1}, \ldots, \beta_{q+2}\right) \in \widetilde{A}(\gamma, N), \beta_{1}+\cdots+\beta_{q+2}=k} P_{\beta_{1}}^{\left(\gamma_{1}\right)}[N, \vec{u}(x, t)] P_{\beta_{2}}^{\left(\gamma_{2}\right)}\left[N, \vec{u}\left(\eta_{1}, t\right)\right] \cdots \\
\quad \times P_{\beta_{q+1}}^{\left(\gamma_{q+1}\right)}\left[N, \vec{u}\left(\eta_{q}, t\right)\right] P_{\beta_{q+2}}^{\left(\gamma_{q+2}\right)}\left[N, \vec{u}^{\prime}(x, t)\right] \\
\widetilde{A}(\gamma, N)=\left\{\left(\beta_{1}, \ldots, \beta_{q+2}\right) \in \mathbb{Z}_{+}^{q+2}: \gamma_{i} \leq \beta_{i} \leq N \gamma_{i}, 1 \leq i \leq q+2\right\} .
\end{array}\right.
$$

We deduce from (4.10), (4.13) that

$$
\begin{aligned}
f[h] & =f\left[u_{0}\right]+\sum_{1 \leq|\gamma| \leq N} \frac{1}{\gamma !} D^{\gamma} f\left[u_{0}\right] \sum_{k=|\gamma|}^{N} \Psi_{k}\left[\gamma, N, \vec{u}, \vec{u}^{\prime}\right] \varepsilon^{k}+|\varepsilon|^{N+1} \hat{R}_{N}\left[f, u_{0}, \vec{u}, \vec{u}^{\prime}, \varepsilon\right] \\
& =f\left[u_{0}\right]+\sum_{k=1}^{N}\left(\sum_{1 \leq|\gamma| \leq k} \frac{1}{\gamma !} D^{\gamma} f\left[u_{0}\right] \Psi_{k}\left[\gamma, N, \vec{u}, \vec{u}^{\prime}\right]\right) \varepsilon^{k}+|\varepsilon|^{N+1} \hat{R}_{N}\left[f, u_{0}, \vec{u}, \vec{u}^{\prime}, \varepsilon\right] \\
& =f\left[u_{0}\right]+\sum_{k=1}^{N} \bar{\Phi}_{k}[N, f] \varepsilon^{k}+|\varepsilon|^{N+1} \hat{R}_{N}\left[f, u_{0}, \vec{u}, \vec{u}^{\prime}, \varepsilon\right]
\end{aligned}
$$

where $\bar{\Phi}_{k}[N, f], 1 \leq k \leq N$, are defined by (4.4)-(4.6) and

$$
\begin{aligned}
& |\varepsilon|^{N+1} \hat{R}_{N}\left[f, u_{0}, \vec{u}, \vec{u}^{\prime}, \varepsilon\right] \\
& \quad=\sum_{1 \leq|\gamma| \leq N} \frac{1}{\gamma !} D^{\gamma} f\left[u_{0}\right] \sum_{k=N+1}^{|\gamma| N} \Psi_{k}\left[\gamma, N, \vec{u}, \vec{u}^{\prime}\right] \varepsilon^{k}+|\varepsilon|^{N+1} R_{N}^{(1)}\left[f, u_{0}, h_{1}, \varepsilon\right] .
\end{aligned}
$$

By the boundedness of the functions $u_{k}, \dot{u}_{k}, 1 \leq k \leq N$, in the function space $L^{\infty}(0, T$; $\left.H^{1}\right)$, we obtain from (4.11), (4.14), and (4.16) that $\left\|\hat{R}_{N}\left[f, u_{0}, \vec{u}, \vec{u}^{\prime}, \varepsilon\right]\right\|_{L^{\infty}\left(0, T ; L^{2}\right)} \leq C$, where $C$ is a constant depending only on $N, T, f, u_{k}, \dot{u}_{k}, 1 \leq k \leq N$. Thus, Lemma 4.3 is proved.

Remark 4.2 Lemma 4.3 is a generalization of the formula contained in ([7], p.262, formula (4.38)) and then Lemma 4.4 follows. These lemmas are the key to establishing the 
asymptotic expansion of the weak solution $u_{\varepsilon}$ of order $N+1$ in a small parameter $\varepsilon$ as below.

Let $u=u_{\varepsilon} \in W_{1}(M, T)$ be the unique weak solution of the problem $\left(P_{\varepsilon}\right)$. Then $v=u_{\varepsilon}-$ $\sum_{k=0}^{N} u_{k} \varepsilon^{k} \equiv u_{\varepsilon}-h$ satisfies the problem

$$
\left\{\begin{aligned}
v^{\prime \prime}-\Delta v= & f[v+h]-f[h]+\varepsilon\left(f_{1}[v+h]-f_{1}[h]\right) \\
& +E_{\varepsilon}(x, t), \quad 0<x<1,0<t<T, \\
v_{x}(0, t)- & h_{0} v(0, t)=v_{x}(1, t)+h_{1} v(1, t)=0, \\
v(x, 0)= & v^{\prime}(x, 0)=0,
\end{aligned}\right.
$$

where

$$
E_{\varepsilon}(x, t)=f[h]-f\left[u_{0}\right]+\varepsilon f_{1}[h]-\sum_{k=1}^{N} F_{k} \varepsilon^{k},
$$

and $F_{k}, 1 \leq k \leq N$, are defined by formulas (4.3).

Then we have the following lemma.

Lemma 4.4 Let $\left(H_{1}\right)$ and $\left(H_{2}^{(N)}\right)$ hold. Then there exists a constant $C_{*}$ such that

$$
\left\|E_{\varepsilon}\right\|_{L^{\infty}\left(0, T ; L^{2}\right)} \leq C_{*}|\varepsilon|^{N+1}
$$

where $C_{*}$ is a constant depending only on $N, T, f, f_{1}, u_{k}, 0 \leq k \leq N$.

Proof of Lemma 4.4 In the case of $N=1$, the proof of Lemma 4.4 is easy, hence we omit the details, which we only prove with $N \geq 2$.

By using formula (4.8) for the function $f_{1}[h]$, we obtain

$$
f_{1}[h]=f_{1}\left[u_{0}\right]+\sum_{k=1}^{N-1} \bar{\Phi}_{k}\left[N-1, f_{1}\right] \varepsilon^{k}+|\varepsilon|^{N} \hat{R}_{N-1}\left[f_{1}, u_{0}, \vec{u}, \vec{u}^{\prime}, \varepsilon\right],
$$

where $\left\|\hat{R}_{N-1}\left[f_{1}, u_{0}, \vec{u}, \vec{u}^{\prime}, \varepsilon\right]\right\|_{L^{\infty}\left(0, T ; L^{2}\right)} \leq C$, with $C$ is a constant depending only on $N, T, f_{1}$, $u_{k}, 0 \leq k \leq N$.

By (4.20), we rewrite $\varepsilon f_{1}[h]$ as follows:

$$
\varepsilon f_{1}[h]=\varepsilon f_{1}\left[u_{0}\right]+\sum_{k=2}^{N} \bar{\Phi}_{k-1}\left[N-1, f_{1}\right] \varepsilon^{k}+\varepsilon|\varepsilon|^{N} \hat{R}_{N-1}\left[f_{1}, u_{0}, \vec{u}, \vec{u}^{\prime}, \varepsilon\right] .
$$

Hence, we deduce from (4.8) and (4.21) that

$$
\begin{aligned}
f[h] & -f\left[u_{0}\right]+\varepsilon f_{1}[h] \\
= & \left(f_{1}\left[u_{0}\right]+\bar{\Phi}_{1}[N, f]\right) \varepsilon+\sum_{k=2}^{N}\left(\bar{\Phi}_{k}[N, f]+\bar{\Phi}_{k-1}\left[N-1, f_{1}\right]\right) \varepsilon^{k} \\
& \quad+|\varepsilon|^{N+1} \tilde{R}_{N}\left[f, f_{1}, u_{0}, \vec{u}, \vec{u}^{\prime}, \varepsilon\right],
\end{aligned}
$$


where

$$
|\varepsilon|^{N+1} \tilde{R}_{N}\left[f, f_{1}, u_{0}, \vec{u}, \vec{u}^{\prime}, \varepsilon\right]=|\varepsilon|^{N+1} \hat{R}_{N}\left[f, u_{0}, \vec{u}, \vec{u}^{\prime}, \varepsilon\right]+\varepsilon|\varepsilon|^{N} \hat{R}_{N-1}\left[f_{1}, u_{0}, \vec{u}, \vec{u}^{\prime}, \varepsilon\right] .
$$

Combining (4.3), (4.18), and (4.22) leads to

$$
E_{\varepsilon}(x, t)=|\varepsilon|^{N+1} \tilde{R}_{N}\left[f, f_{1}, u_{0}, \vec{u}, \vec{u}^{\prime}, \varepsilon\right]
$$

By the boundedness of the functions $u_{k}, u_{k}^{\prime}, 0 \leq k \leq N$, in the function space $L^{\infty}(0, T$; $H^{1}$ ), we obtain from (4.8), (4.20), (4.23), and (4.24) that

$$
\left\|E_{\varepsilon}\right\|_{L^{\infty}\left(0, T ; L^{2}\right)} \leq C_{*}|\varepsilon|^{N+1}
$$

where $C_{*}$ is a constant depending only on $N, T, f, f_{1}, u_{k}, u_{k}^{\prime}, 0 \leq k \leq N$.

The proof of Lemma 4.4 is complete.

Proof of Theorem 4.2 Consider the sequence $\left\{v_{m}\right\}$ defined by

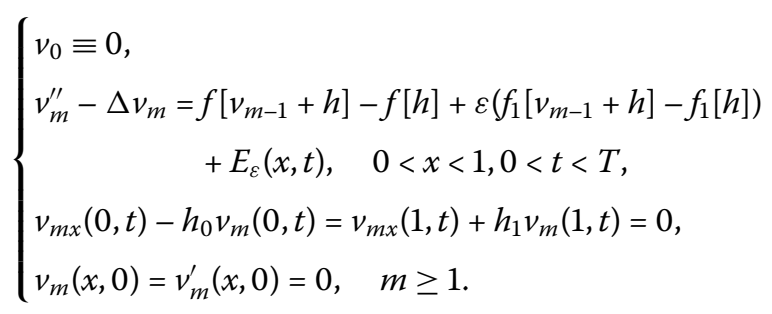

By multiplying two sides of (4.26) with $v_{m}^{\prime}$ and after integration in $t$, we have

$$
\begin{aligned}
Z_{m}(t)= & 2 \int_{0}^{t}\left\langle E_{\varepsilon}(s), v_{m}^{\prime}(s)\right\rangle d s+2 \int_{0}^{t}\left\langle f\left[v_{m-1}+h\right]-f[h], v_{m}^{\prime}(s)\right\rangle d s \\
& +2 \varepsilon \int_{0}^{t}\left\langle f_{1}\left[v_{m-1}+h\right]-f_{1}[h], v_{m}^{\prime}(s)\right\rangle d s \\
= & \bar{J}_{1}+\bar{J}_{2}+\bar{J}_{3},
\end{aligned}
$$

where $Z_{m}(t)=\left\|v_{m}^{\prime}(t)\right\|^{2}+\left\|v_{m}(t)\right\|_{a}^{2}$.

We estimate the integrals on the right-hand side of (4.27) as follows.

Estimating $\bar{J}_{1}$. By using Lemma 4.4, we deduce that

$$
\bar{J}_{1}=2 \int_{0}^{t}\left\langle E_{\varepsilon}(s), v_{m}^{\prime}(s)\right\rangle d s \leq T C_{*}^{2}|\varepsilon|^{2 N+2}+\int_{0}^{t} Z_{m}(s) d s .
$$

Estimating $\bar{J}_{2}$. We note that

$$
\left\|f\left[v_{m-1}+h\right]-f[h]\right\| \leq \sqrt{2}(q+1) K_{M_{*}}(f)\left\|v_{m-1}\right\|_{W_{1}(T)},
$$

with $M_{*}=(N+2) M$. 
It follows from (4.29) that

$$
\begin{aligned}
\bar{J}_{2} & \leq 2 \int_{0}^{t}\left\|f\left[v_{m-1}+h\right]-f[h]\right\|\left\|v_{m}^{\prime}(s)\right\| d s \\
& \leq 2 T(q+1)^{2} K_{M_{*}}^{2}(f)\left\|v_{m-1}\right\|_{W_{1}(T)}^{2}+\int_{0}^{t} Z_{m}(s) d s .
\end{aligned}
$$

Estimating $\bar{J}_{3}$. Similarly,

$$
\begin{aligned}
\bar{J}_{3} & \leq 2 \int_{0}^{t}\left\|f_{1}\left[v_{m-1}+h\right]-f_{1}[h]\right\|\left\|v_{m}^{\prime}(s)\right\| d s \\
& \leq 2 T(q+1)^{2} K_{M_{*}}^{2}\left(f_{1}\right)\left\|v_{m-1}\right\|_{W_{1}(T)}^{2}+\int_{0}^{t} Z_{m}(s) d s .
\end{aligned}
$$

Combining (4.27), (4.28), (4.30), and (4.31) leads to

$$
\begin{aligned}
Z_{m}(t) \leq & 2 T(q+1)^{2}\left[K_{M_{*}}^{2}(f)+K_{M_{*}}^{2}\left(f_{1}\right)\right]\left\|v_{m-1}\right\|_{W_{1}(T)}^{2} \\
& +T C_{*}^{2}|\varepsilon|^{2 N+2}+3 \int_{0}^{t} Z_{m}(s) d s
\end{aligned}
$$

By using Gronwall's lemma, we deduce from (4.32) that

$$
\left\|v_{m}\right\|_{W_{1}(T)} \leq \sigma_{T}\left\|v_{m-1}\right\|_{W_{1}(T)}+\delta_{T}(\varepsilon), \quad \text { for all } m \geq 1,
$$

where

$$
\begin{aligned}
& \sigma_{T}=\sqrt{2}(q+1)\left(1+\frac{1}{\sqrt{a_{0}}}\right) \sqrt{K_{M_{*}}^{2}(f)+K_{M_{*}}^{2}\left(f_{1}\right)} \sqrt{T e^{3 T}}, \\
& \delta_{T}(\varepsilon)=C_{*}\left(1+\frac{1}{\sqrt{a_{0}}}\right) \sqrt{T e^{3 T}}|\varepsilon|^{N+1} .
\end{aligned}
$$

We can assume that

$$
\sigma_{T}<1, \quad \text { with the suitable constant } T>0 \text {. }
$$

We require the following lemma whose proof is immediate.

Lemma 4.5 Let the sequence $\left\{\gamma_{m}\right\}$ satisfy

$$
\gamma_{m} \leq \sigma \gamma_{m-1}+\delta \quad \text { for all } m \geq 1, \quad \gamma_{0}=0
$$

where $0 \leq \sigma<1, \delta \geq 0$ are the given constants. Then

$$
\gamma_{m} \leq \delta /(1-\sigma) \quad \text { for all } m \geq 1
$$

Applying Lemma 4.5 with $\gamma_{m}=\left\|v_{m}\right\|_{W_{1}(T)}, \sigma=\sigma_{T}<1, \delta=\delta_{T}(\varepsilon)$, it follows from (4.36) that

$$
\left\|v_{m}\right\|_{W_{1}(T)} \leq \frac{\delta_{T}(\varepsilon)}{1-\sigma_{T}}=C_{T}|\varepsilon|^{N+1},
$$

where $C_{T}$ is a constant depending only on $T$. 
On the other hand, the linear recurrent sequence $\left\{v_{m}\right\}$ defined by (4.26) converges strongly in the space $W_{1}(T)$ to the solution $v$ of problem (4.17). Hence, letting $m \rightarrow+\infty$ in (4.37), we get

$$
\|v\|_{W_{1}(T)} \leq C_{T}|\varepsilon|^{N+1}
$$

This implies (4.7). The proof of Theorem 4.2 is complete.

\section{Acknowledgements}

The authors wish to thank the referee for their valuable criticism and suggestions, leading to the present improved version of our paper. There are no funding sources supporting this work.

\section{Competing interests}

The authors declare that there is no conflict of interests regarding the publication of this paper.

\section{Authors' contributions}

All authors contributed equally to the writing of this paper. All authors read and approved the final manuscript.

\section{Author details}

${ }^{1}$ Dong Nai University, 4 Le Quy Don Str., Tan Hiep District, Bien Hoa City, Vietnam. ${ }^{2}$ Department of Mathematics and Computer Science, VNUHCM - University of Science, 227 Nguyen Van Cu Str., Dist. 5, Ho Chi Minh City, Vietnam. ${ }^{3}$ Khanh Hoa University, 01 Nguyen Chanh Str., Nha Trang City, Vietnam.

\section{Publisher's Note}

Springer Nature remains neutral with regard to jurisdictional claims in published maps and institutional affiliations.

Received: 13 September 2016 Accepted: 26 May 2017 Published online: 07 June 2017

\section{References}

1. Beilin, SA: On a mixed nonlocal problem for a wave equation. Electron. J. Differ. Equ. 2006, 103 (2006)

2. Bergounioux, M, Long, NT, Dinh, APN: Mathematical model for a shock problem involving a linear viscoelastic bar. Nonlinear Anal. 43, 547-561 (2001)

3. Benaissa, A, Messaoudi, SA: Exponential decay of solutions of a nonlinearly damped wave equation. Nonlinear Differ. Equ. Appl. 12, 391-399 (2005)

4. Jokhadze, O: The global Cauchy problem for wave equations with nonlinear damping term. Differ. Equ. 50(1), 57-65 (2014)

5. Ngoc, LTP, Hang, LNK, Long, NT: On a nonlinear wave equation associated with the boundary conditions involving convolution. Nonlinear Anal. TMA 70(11), 3943-3965 (2009)

6. Long, NT, Diem, TN: On the nonlinear wave equation $u_{t t}-u_{x x}=f\left(x, t, u, u_{x}, u_{t}\right)$ associated with the mixed homogeneous conditions. Nonlinear Anal. TMA 29(11), 1217-1230 (1997)

7. Long, NT: On the nonlinear wave equation $u_{t t}-B\left(t,\|u\|^{2},\left\|u_{x}\right\|^{2}\right) u_{x x}=f\left(x, t, u, u_{x}, u_{t},\|u\|^{2},\left\|u_{x}\right\|^{2}\right)$ associated with the mixed homogeneous conditions. J. Math. Anal. Appl. 306(1), 243-268 (2005)

8. Long, NT, Ngoc, LTP: On a nonlinear wave equation with boundary conditions of two-point type. J. Math. Anal. Appl. 385(2), 1070-1093 (2012)

9. Ngoc, LTP, Long, NT: Existence and exponential decay for a nonlinear wave equation with a nonlocal boundary condition. Commun. Pure Appl. Anal. 12(5), 2001-2029 (2013)

10. Truong, LX, Ngoc, LTP, Dinh, APN, Long, NT: The regularity and exponential decay of solution for a linear wave equation associated with two-point boundary conditions. Nonlinear Anal., Real World Appl. 11(1-2), 1289-1303 (2010)

11. Bhattarai, S: Stability of solitary-wave solutions of coupled NLS equations with power-type nonlinearities. Adv Nonlinear Anal. 4(2), 73-90 (2015)

12. Kumar, S: Fractional modelling arising in unidirectional propagation of long waves in dispersive media. Adv Nonlinear Anal. 5(4), 383-394 (2016). doi:10.1515/anona-2013-0033

13. Repovš, D: Stationary waves of Schrödinger-type equations with variable exponent. Anal. Appl. 13(6), 645-661 (2015) doi:10.1142/S0219530514500420

14. Pellicer, M, Solà-Morales, J: Spectral analysis and limit behaviours in a spring-mass system. Commun. Pure Appl. Math. 7(3), 563-577 (2008)

15. Brezis, H: Functional Analysis, Sobolev Spaces and Partial Differential Equations. Springer, New York (2010)

16. Showater, RE: Hilbert space methods for partial differential equations. Electron. J. Differ. Equ. Monograph 01 (1994)

17. Lions, JL: Quelques méthodes de résolution des problèmes aux limites nonlinéaires. Dunod, Paris (1969) 